\title{
Is the Mexican wave really a ripple of excitation?
}

Julyan H. E. Cartwright,

Laboratorio de Estudios Cristalográficos, CSIC • E-18100 Granada • Spain.

$\mathrm{T}$ he World Cup has come and gone once again, and with it we have seen more examples of that phenomenon of mass audience participation in stadia - first brought to the world's attention during the 1986 World Cup in Mexico - the Mexican Wave. In the pages of Nature at the time of the last World Cup in 2002 an analysis of the Wave appeared that suggested that it is an excitable phenomenon [1]. An excitable element is defined by its response to a perturbation: whereas a small disturbance causes merely an equally small response, a perturbation above a certain threshold in amplitude excites a quiescent element that then decays back to quiescence during a refractory period in which it is unresponsive to further excitation. Such elements, when coupled to their neighbours into an assembly, become an excitable medium. Forest fires, plankton populations, and the heart are just a few of the physical systems that can be thus described. Waves of excitation can propagate through excitable media; that is, like heart fibrillations, or forest fires, a wave sweeps through the medium and activates quiescent excitable elements, that then go through an unresponsive refractory period before becoming excitable once more.

People are certainly excited when they take part in the Wave, but are they excitable elements in the scientific sense of the term? To answer this question we may ask ourselves what would happen if two Mexican Waves were set off in opposite

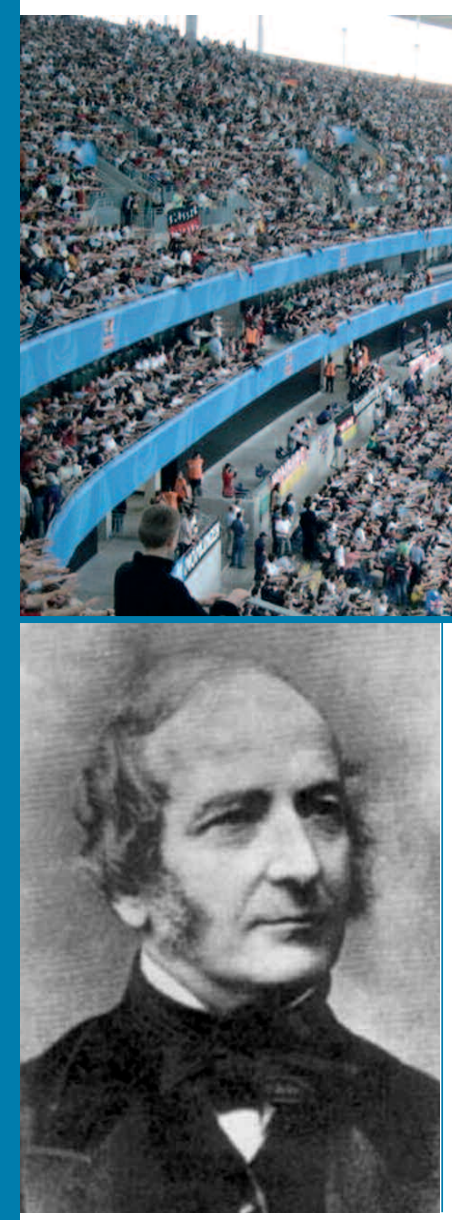

A Fig. 1: A Mexican Wave at the Confederations Cup, 2005 (image taken by Florian K. courtesy of Wikipedia under GNU Free Documentation licence)

- Fig. 2: Forest fires may be modelled as excitable systems; after this fire has extinguished itself, another cannot pass until the vegetation has regrown (image taken by John McColgan courtesy of Wikipedia)

4 Fig. 3: John Scott Russell directions around a stadium - would they mutually annihilate on meeting, as excitable waves do? This is a crucial test, since one characteristic of an excitable medium is the refractory period during which excitation is impossible. In a forest, a second fire cannot pass until the vegetation burnt by the the first has grown back, and cardiac cells cannot fire again until they have recovered from their first firing. In other, nonexcitable, media lacking this refractory period, waves pass through each other rather than destroying each other. It's not obvious that there should be a refractory period involved in the Mexican Wave, or at least the limitation wouldn't seem to be physiological; there's no apparent reason why, having just sat down, wavers shouldn't jump back on their feet again for another wave coming from the other direction.

Nowadays, with the Internet, it is easier than it used to be to answer this type of question; masses of relevant data are just an Internet search away, and one often doesn't have to perform the experiment oneself - the results are already out there. And so it proved in this instance; the Wikipedia web page on the "audience wave" [2] contains the information sought for: "Simultaneous, counter-rotating waves have been produced", it informs us. The text goes on to note that one of these cases occurred at the Sydney Olympic Games in 2000, and also that "in non-circular seating arrangements, the wave can instead reflect back and forth through the audience". This is another reason to doubt that the Wave is excitable; unlike other waves, excitable waves do not reflect from boundaries.

So what are Mexican waves if not excitable? Similar solitary waves like these that propagate without dispersing and pass through each other unscathed are found in many nonlinear media, and are often termed solitons. Nowadays solitons are used commercially in optical communications technology, but the archetypal examples are the shallow-water waves like that famously seen by their discoverer John Scott Russell on the Union canal near Edinburgh: "The mass of water ... rolled forward ... assuming the shape of a large solitary elevation. I followed it on horseback, and overtook it still rolling on at a rate of some eight or nine miles an hour ... [until] I lost it in the windings of the channel. Such, in the month of August 1834, was my first chance interview with that singular and beautiful phenomenon" [3]. From such beginnings in

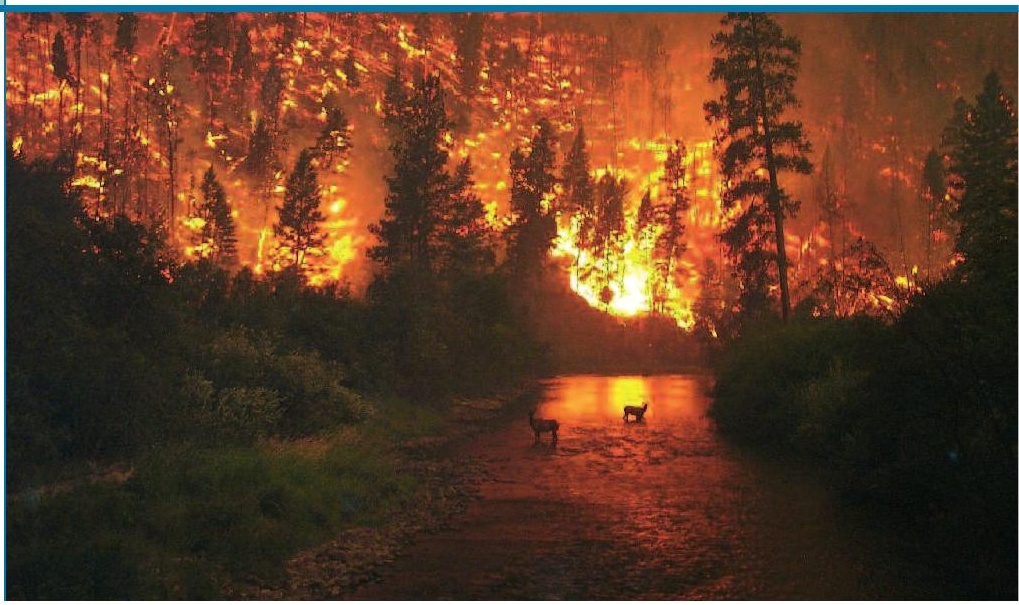




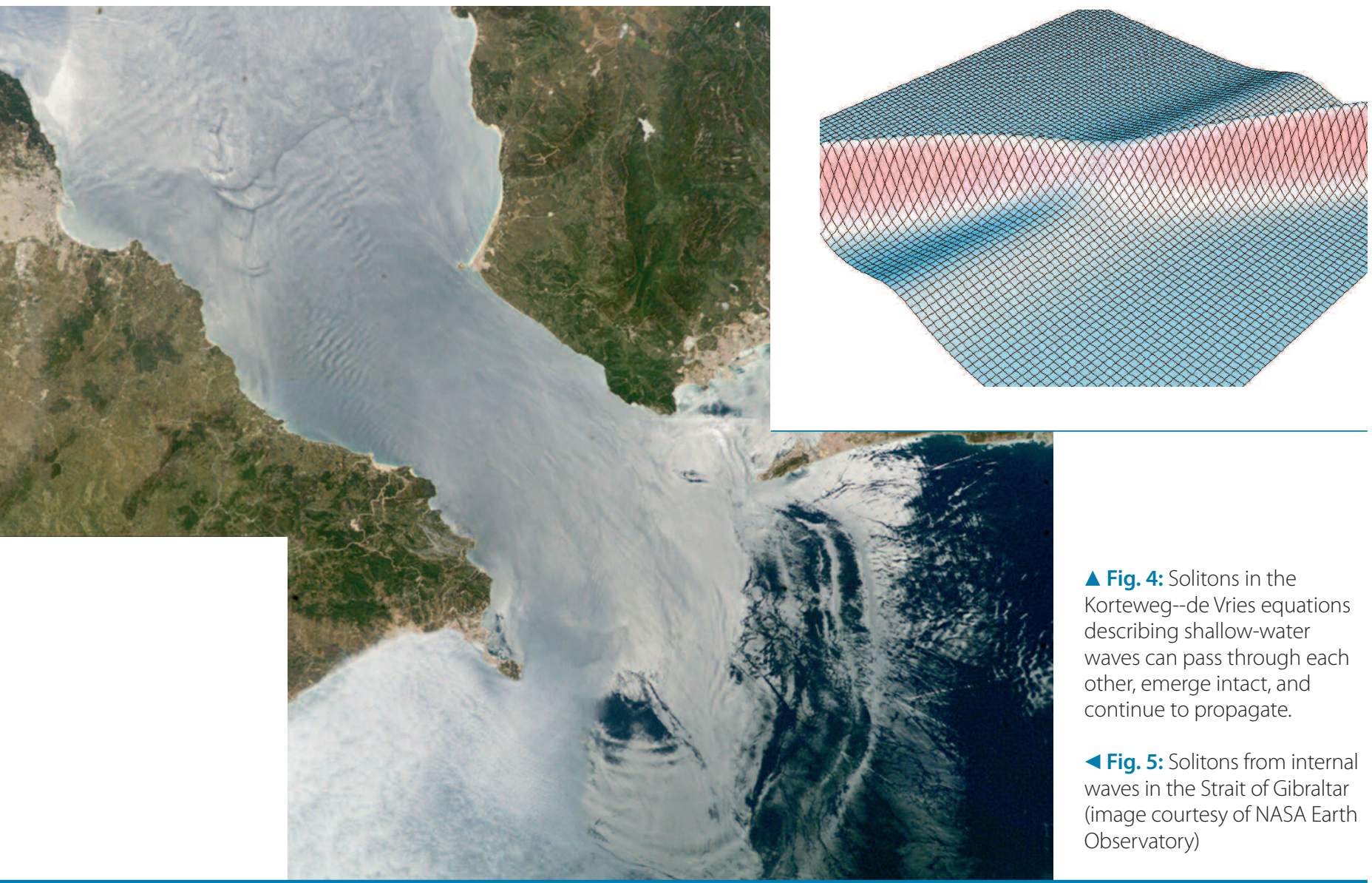

the nineteenth century, shallow-water solitons have latterly been implicated in less romantic phenomena such as large waves created by fast ships on shallow lakes and seas that can swamp small boats, and even larger waves, tsunami, for which the oceans themselves are shallow waters. Solitons exist in shallow water because it is a nonlinear medium - only a train of waves is stable in a linear medium, while a solitary wave disperses there because it is not stable - and similar solitons in the nonlinear medium of an optical fibre are exploited to transmit information over huge distances without loss. Mexican Waves then are better described as solitons rather than excitable waves.

The stadium crowd is a macroscopically discrete medium and not a continuum one such as water, but acts as a discretization of a nonlinear medium in which solitary waves can travel like in the shallow water of a canal. Of course, both solitons and excitable waves are the inevitable results of the dynamics of their underlying media, whether that is water, combustible vegetation, or cardiac tissue, while Mexican Waves are people having fun. People can choose whether or not to stand up and be part of a Wave, and Mexican Waves happen because most people present want to make a wave, rather than nothing at all, or something much more complicated; or rather than sitting back and watching the sport! So we shouldn't expect a set of strict rules to underlie Mexican Waves; rather heuristic principles. The underlying rule for being part of a Wave is like that we use at the beach, when we play the game of jumping through the incoming waves. It goes something like: "when a wave approaches me, get ready and jump up as it arrives".

As so often happens in science, from our initial examination of an apparently trivial phenomenon, the Mexican Wave, we can now begin to extract some worthwhile conclusions. On one hand, we are led to contemplate the ubiquity of wave phenomena in nonlinear media, both continuous and discrete; an excitable medium is just one instance of this, and is not the best description of the stadium crowd. On the other, we can start to see how complex human behaviour might be modelled through applying heuristic rules such as those we have described above. There is much to be learned through studying the new field of what has been called sociophysics with interacting agents that function by such rules [4]. It is fascinating to see how, both with Mexican Waves and in other cases, similar types of phenomena emerge from interacting intelligent agents as from the inaminate matter traditionally studied in other areas of physics. Scientifically then Mexican Waves may be described as solitons; we may say that they are exciting, but not excitable.

\section{About the author:}

Julyan Cartwright is with the Spanish Consejo Superior de Investigaciones Científicas (CSIC), and is based at the Laboratory for Crystallographic Studies in Granada, Spain. He may be contacted at julyan@lec.csic.es

\section{References}

[1] I. Farkas, D. Helbing, and T. Vicsek. Social behaviour: Mexican waves in an excitable medium. Nature, 419:131-132, 2002.

[2] http://en.wikipedia.org/wiki/Audience wave.

[3] J. Scott Russell. Report on waves'. In Proceedings of the $14^{\text {th }}$ meeting of the BAAS, pages 311-390, 1844.

[4] P. Ball. Critical Mass: How One Thing Leads to Another. Random House, 2004 